\title{
Sistemas personalizados de dosificación como herramienta para el cambio de actitud hacia los medicamentos en una paciente joven
}

\author{
Ángel Soliño Comedeiro', Rocío Portal Dávila², Elena Touriño Baliña², Lucía Aragunde Álvarez², \\ Patricia Gómez Mariño² \\ 1. Farmacéutico comunitario en Vigo. 2. Farmacéutica comunitaria en Vigo.
}

\section{PALABRAS CLAVE}

Servicios comunitarios de farmacia, servicios farmacéuticos, cumplimiento de la medicación, calidad de vida, conductas de riesgo para la salud, experiencia farmacoterapéutica, problemas farmacoterapéuticos, gestión integral de la farmacoterapia, prevención del suicidio, sistema personalizado de dosificación

\section{ABREVIATURAS}

SPD: sistemas personalizados de dosificación

SPF: servicio profesional

farmacéutico

\section{KEYWORDS}

Community pharmacy services, pharmaceutical services, medication adherence, quality of life, health risk behaviors, medication experiences, drug therapy problems, medication therapy management, suicide prevention, multicompartment compliance aids

\section{Introducción}

Los sistemas personalizados de dosificación (SPD) constituyen una herramienta útil para la mejora de la adherencia terapéutica y suponen para el farmacéutico comunitario una oportunidad como profesional sanitario para asumir responsabilidades hacia sus pacientes (1).

Habitualmente, la población diana del servicio profesional farmacéutico (SPF) de SPD incluye ancianos, personas con problemas cognitivos, polimedicados o pacientes que deben utilizar medicamentos con pautas complejas (2). Sin embargo, el SPD puede utilizarse en muchos más casos con el objetivo de ayudar a las personas que utilizan medicamentos a mejorar su calidad de vida.

Tabla 1 Medicación prescrita e indicaciones
Galicia es una de las comunidades autónomas con mayor tasa de suicidio juvenil en España. Se trata de un problema multifactorial donde caben destacar como factores de riesgo los trastornos depresivos y de adaptación, así como la disponibilidad de métodos suicidas (3). Existe un predominio de suicidios entre la población masculina, mientras que las tentativas de suicidio se producen mayoritariamente entre la población femenina, siendo la sobredosificación de medicamentos el método más habitual (4).

\section{Descripción del caso}

Mujer de 23 años, estudiante, con diagnóstico principal de depresión y polimedicación moderada (tabla 1).

\begin{tabular}{|c|l|l|c|l|}
\hline $\begin{array}{c}\text { Código } \\
\text { Nacional }\end{array}$ & Medicamento & \multicolumn{1}{|c|}{ Principio activo } & Posología & Indicación \\
\hline 668434 & $\begin{array}{l}\text { Seropram } \\
30 \mathrm{mg}\end{array}$ & Citalopram & $1-0-0$ & Depresión \\
\hline 662387 & $\begin{array}{l}\text { Norebox } \\
4 \mathrm{mg}\end{array}$ & Reboxetina & $1-0-0$ & Depresión \\
\hline 830836 & $\begin{array}{l}\text { Surmontil } \\
25 \mathrm{mg}\end{array}$ & Trimipramina & $0-0-2$ & Depresión \\
\hline 855304 & $\begin{array}{l}\text { Diane Diario } \\
2 \mathrm{mg} / 35 \mathrm{mcg}\end{array}$ & Ciproterona/Etinilestradiol & $0-0-1$ & Virilización \\
\hline 703074 & $\begin{array}{l}\text { Aldoleo } \\
50 \mathrm{mg} / 50 \mathrm{mg}\end{array}$ & Clortalidona/Espironolactona & $1-0-0$ & Edema \\
\hline
\end{tabular}

* Medicación prescrita al momento de la incorporación al SPF de SPD y confirmada con el médico de referencia de la paciente.

Este trabajo fue premiado en la categoría CASOS de la 2a edición de los Premios SEFAC-Fundación Mylan para la Salud a la mejora de la adherencia terapéutica mediante el uso de SPD en farmacias comunitarias españolas que fue entregado durante el $40^{\circ}$ Congreso Nacional de Semergen en octubre de 2018. como herramienta para el cambio de actitud hacia los medicamentos en una paciente joven. Farmacéuticos Comunitarios. 2019 Sep 30;11(3):19-21. doi:10.5672/FC.2173-9218.(2019/Nol11).003.04 
La paciente se traslada desde su núcleo familiar a una nueva ciudad para continuar sus estudios universitarios, donde convive con otros estudiantes.

En su nuevo entorno se encuentra un ambiente hostil que junto con su diagnóstico de depresión propician una tentativa de suicidio utilizando benzodiacepinas.

A raiz de este suceso los medicamentos adquieren un nuevo significado de peligro y se transforman en un motivo de angustia tanto para la paciente como para su familia. Esto hace que se planteen el retorno de la paciente a su hogar donde dispondría del apoyo familiar; como alternativa, buscan soluciones que permitan gestionar su medicación de forma adecuada sin que sea necesario interrumpir sus estudios universitarios.

La paciente y su madre acuden a una farmacia buscando posibles soluciones. Desde ahí la derivan a nuestra farmacia para su incorporación a la unidad de SPD, dado que ellos no disponen de este SPF.

Se concierta una entrevista a la que acude también su madre, en quien la paciente delega parte de la responsabilidad. Recogemos la información relativa a su farmacoterapia y su experiencia farmacoterapéutica $(5,6)$.

La primera opción que se plantea es la realización de un SPD estándar, siguiendo los protocolos establecidos (2), donde la medicación queda custodiada en la farmacia y se realizan entregas en blísteres semanales.

Sin embargo, dado que el caso no se ajusta a un perfil tipo de SPD, y teniendo en cuenta al gran peso que aquí tiene la experiencia farmacoterapéutica, se entiende que un SPD tipo tampoco satisfaría las necesidades de la paciente. A todo esto, se suma que tanto ella como su madre consideran que la disponibilidad en el domicilio de la medicación para una semana todavía supone un elemento peligroso, a pesar de que realmente no existe un riesgo mortal con esa cantidad de medicación.

En consecuencia, surge la necesidad de buscar nuevas soluciones que aseguren el uso correcto de los medicamentos, minimicen el riesgo de abuso y mejoren su calidad de vida.

Atendiendo a todas las circunstancias particulares del caso se decide utilizar la Guía de Apoyo Decisional de Ottawa $(7,8)$ como herramienta para la toma de decisiones compartidas con la paciente y su entorno familiar. Esta guía permite explorar las opciones disponibles como ayuda para las personas que enfrentan decisiones en el ámbito sociosanitario.

Finalmente, se acuerda establecer un estrecho contacto telefónico tanto con ella como con su madre, designada como persona de contacto ante cualquier incidencia, así como la preparación de un SPD adaptado, donde la medicación queda custodiada en la farmacia y se entrega en tres blísteres semanales independientes de la siguiente manera:

- A recoger el lunes por la tarde: medicación correspondiente a lunes por la noche, martes y miércoles por la mañana.

- A recoger el miércoles por la tarde: medicación correspondiente a miércoles por la noche, jueves y viernes por la mañana.

- A recoger el viernes por la tarde: medicación correspondiente a viernes por la noche, sábado, domingo y lunes por la mañana.

También se establecen visitas de seguimiento (9) con la paciente para atender todas sus necesidades farmacoterapéuticas de forma global y con el objetivo de establecer y afianzar una relación terapéutica basada en la confianza.

\section{Resultados}

La custodia de la medicación y la elaboración de los SPD permitieron que se hiciese un uso adecuado y seguro de la misma. Además, se eliminó la percepción de la medicación como elemento agresor tanto para la paciente como para su entorno familiar. Esto supuso una gran mejora en la calidad de vida de ambas partes.

Además, el sistema de tres recogidas semanales junto con las visitas de seguimiento permitió fortalecer la relación terapéutica basada en la confianza establecida entre los profesionales sanitarios y la paciente. A lo largo de dichas visitas nos reveló los problemas que tenía en su entorno universitario y personal, desvelando sus temores y su sensación de inseguridad en sí misma, lo que nos permitió conocer mejor su experiencia con la medicación. Esto se evidenció cuando, tras 4 meses de seguimiento, la paciente consiguió sin receta en otra farmacia un envase de Noctamid $2 \mathrm{mg}$ (Lormetazepam) planeando una nueva tentativa de suicidio que nunca llegó a ejecutar, pues finalmente decidió entregárnosla diciendo "prefiero que la tengáis vosotros".

\section{Discusión}

A priori nadie consideraría a esta paciente, joven y universitaria, susceptible de utilizar un SPD. No es el paciente anciano, polimedicado y con problemas cognitivos que habitualmente se considera como paciente tipo para recibir un SPD. Esta utilidad, en un paciente distinto del habitual, se basa en la mejora de la calidad de vida en casos cuya experiencia farmacoterapéutica requiera intervención y seguimiento por parte de profesionales con una visión integradora que no se limite a perseguir solamente la adherencia terapéutica y que entiendan a los pacientes como personas que utilizan medicamentos inmersos en su propia y compleja realidad.

Además, es destacable que no sólo se mejora la calidad de vida del paciente, sino también la de su familia al reducir los riesgos existentes en su entorno.

Gracias a todo esto se facilita un adecuado control de los problemas de salud y, en último término, la conservación de la vida.

Por último, debemos remarcar que, si consideramos el SPD de forma aislada, sin el complemento imprescindible del seguimiento farmacoterapéutico y el establecimiento de una relación terapéutica entre los profesionales sanitarios y la paciente, la utilidad de dicha herramienta se verá mermada.

\section{Conclusión}

El SPD demuestra ser una herramienta válida también en casos cuyo perfil difiera del habitual: ancianos, polimedicados, con pautas complejas o con problemas cognitivos.

\section{Referencias bibliográficas}

1. Hepler CD, Strand LM. Oportunidades y responsabilidades en la atención farmacéutica. Pharm Care Esp. 1999; 1(1):35-47.

2. Grupo de Trabajo de Buenas Prácticas del Consejo General de Colegios 
Oficiales de Farmacéuticos. Documento de Buenas Prácticas en Farmacia Comunitaria en España. Servicio de Elaboración y Provisión de Sistemas Personalizados de Dosificación. Consejo General de Colegios Oficiales de Farmacéuticos. 2018. [Internet - consultado el 15 de noviembre de 2018]. Disponible en: https://www.portalfarma.com/Profesionales/Buenas-practicas-profesionales/Documents/BBPP14-SPD.pdf

3. Picazo-Zappino J. El suicidio infanto-juvenil: una revisión. Actas Esp Psiquiatr. 2014; 42(3):125-32.

4. Pérez Camarero S. El suicidio adolescente y juvenil en España. En: Pérez Camarero S, Rojo Mora N, Hidalgo Vega A. La salud mental de las personas jóvenes en España. Madrid: Instituto de la Juventud; 2009. p. 127-142. Disponible en https://consaludmental.
org/publicaciones/Saludmentalpersonasjovenes.pdf\#page $=127$

5. Shoemaker SJ et al. The medication experience: Preliminary evidence of its value for patient education and counseling on chronic medications. Patient Educ. Couns. 2011; 82(3): 443-450. doi:10.1016/j.pec.2011.02.007

6. Ramalho de Oliveira D, Shoemaker SJ, Ekstrand M, Alves MR. Preventing and resolving drug therapy problems by understanding patients' medication experience. J Am Pharm. 2012; 52:7180. doi:10.1331/JAPhA.2012.10239

7. Stacey D, Légaré $\mathrm{F}$, Lewis K, Barry MJ, Bennett CL, Eden KB, Holmes-Rovner M, Llewellyn-Thomas H, Lyddiatt A, Thomson R, Trevena L. Decision aids for people facing health treatment or screening decisions. Cochrane Database Syst Rev. 2017, Issue 4. Art. No.: CD001431. Disponible en: https://
www.cochrane.org/es/CD001431/ayudas-la-toma-de-decisiones-para-pacientes-que-deben-decidir-sobre-tratamientos-o-pruebas-de

8. O’Connor, Stancey, Jacobsen. Guía de Apoyo Decisional de Ottawa. Ottawa Hospital Research Institute and University of Ottawa, Canada. 2012. Traducción española Urrutia 2013. [Internet - consultado el 15 de noviembre de 2018] Disponible en: https://decisionaid.ohri.ca/docs/das/ GAD0.pdf

9. Prats R. coordinador. Dispensación, adherencia y uso adecuado del tratamiento: guía práctica para el farmacéutico comunitario. Madrid: Sociedad Española de Farmacia Familiar y Comunitaria (SEFAC); 2017. ISBN: 978-84697-6252. Disponible en: https://www. sefac.org/sites/default/files/2017-11/ Adherencia_0.pdf 